\title{
Expressão de Afetividade por Agente Pedagógico em Ambiente Virtual de Ensino e Aprendizagem: uma Revisão Sistemática da Literatura
}

Francieli Motter Ludovico - PPGIE - Universidade Federal do Rio Grande do Sul/

Universidade Tecnológica Federal do Paraná - franludovico@ hotmail.com

Adriana Paz Nunes - Universidade Federal do Rio Grande do Sul adriana.paz.nunes@ hotmail.com

Patrícia Campelo Costa Barcellos - PPGIE - Universidade Federal do Rio Grande do Sul - patricia.campelo@ufrgs.br

Resumo: O presente artigo apresenta uma Revisão Sistemática da Literatura (RSL) e propõe análise dos Agentes Pedagógicos (AP) dos estudos selecionados com o objetivo de compreender se a utilização de AP, que expressam afetividade, pode auxiliar nos processos educacionais dentro de Ambientes Virtuais de Ensino e Aprendizagem (AVEA). A RSL foi realizada utilizando as bases do Portal de Periódicos da Capes, na International Conference on Advanced Learning Technologies e na Revista Novas Tecnologias na Educação, e com isso foram encontrados 86 estudos para a revisão, sendo 4 incluídos para a presente análise. O presente estudo evidenciou que a utilização de Agentes Pedagógicos afetivos pode contribuir e influenciar positivamente os processos educacionais que ocorrem em AVEA. Dessa forma, destaca-se a importância de propor reflexões acerca da afetividade na educação mediada por computador.

Palavras-chave: Revisão Sistemática da Literatura; Agente Pedagógico; Expressão de Afetividade; Ambiente Virtual de Ensino e Aprendizagem.

\section{Affective Expression by Pedagogical Agent in Virtual Teaching and Learning Environment: A Systematic Literature Review}

Abstract: This article presents a Literature Systematic Review and proposes an analysis of the Pedagogical Agents (PA) of the selected studies in order to understand if the use of PA, which express affectivity, can help in the educational processes within Teaching and Learning Virtual Environment. The RSL was carried out using the bases of the Capes Journal Portal, the International Conference on Advanced Learning Technologies and Revista Novas Tecnologias na Educação, so that 86 studies were found for the review, with 4 of them included for the present analysis. The study showed that the use of affective Pedagogical Agents can contribute and positively influence the educational processes that occur in the Teaching and Learning Virtual Environment. Thus, the importance of proposing reflection on affectivity in computer-mediated education stands out.

keywords: Literature Systematic Review; Pedagogical Agent; Affectivity Expression; Teaching and Learning Virtual Environment.

\section{1- Introdução}


A afetividade ocupa um espaço importante nos processos educacionais. As práticas e a distância na educação mediada por computador podem isolar o estudante, o que acaba desmotivando-o e, por conseguinte, dificulta seu processo de aprendizagem. Fahy (2008) salienta que o processo de aprendizagem formal não deve ser solitário. Nesse sentido, faz-se necessário refletir sobre caminhos para possibilitar afetividade em Ambientes Virtuais de Ensino e Aprendizagem (AVEA), e os Agentes Pedagógicos (AP) apresentam-se como uma possibilidade para levar a afetividade para dentro desses ambientes, pois são concebidos com a intenção de expressar afetividade, buscando motivar e promover o engajamento do estudante.

O presente estudo traz uma Revisão Sistemática da Literatura para identificar publicações científicas sobre Expressão de Afetividade por Agente Pedagógicos em AVEA, a qual focalizou quatro trabalhos, a partir das regras de exclusão delineadas na seção de metodologia deste trabalho. A partir da seleção foi realizada uma análise dos AP com o objetivo de compreender se sua utilização, que expressa afetividade, pode auxiliar nos processos educacionais dentro de AVEA.

Assim, inicialmente apresenta-se a área de Computação Afetiva, conceitualizando afetividade, emoção, sentimento e Agente Pedagógico, contextualização necessária para auxiliar na compreensão e exploração dos estudos trazidos à discussão. Posteriormente, descreve-se como a Revisão Sistemática da Literatura foi conduzida. Por fim, os trabalhos selecionados são apresentados e analisados.

\section{2- Computação Afetiva, Afetividade e Agentes Pedagógicos}

O termo Computação Afetiva foi cunhado por Rosalind Picard (1997), a qual a define como a área que congrega as pesquisas da aplicabilidade da afetividade em sistemas não-biológicos, ou seja, o uso das emoções em diferentes aspectos nos sistemas computacionais (personalização de interfaces, simulação de sistemas com emoções, pesquisas que envolvem emoções nas interações homem- máquina). De acordo com Bercht (2006, p.106), Computação Afetiva "é um conjunto de técnicas adaptadas da Inteligência Artificial e da Engenharia de Software, agregadas e coordenadas conjuntamente ao estudo, modelamento e simulação da experiência afetiva humana, como característica entremeada e constituidora dos processos cognitivos, orientado a aplicações em domínios bastante variados".

Sendo o homem um sujeito que percebe, raciocina e age sobre o mundo, os sistemas que se relacionam com ele devem apresentar-se como sistemas adaptativos e eficientes, por meio da integração, ou interação, da emoção com a razão. Assim, a afetividade é utilizada com o objetivo de desenvolver sistemas mais inteligentes e mais adaptados ao homem (BERCHT, 2006).

Referente à compreensão do termo afetividade, Bercht (2006) afirma que esse é encontrado na literatura sem uma precisa definição, mas pode-se integrá-lo como todo o domínio das emoções propriamente ditas, dos sentimentos, das emoções, das experiências sensíveis e, principalmente, da capacidade em se poder entrar em contato com sensações.

Nesse sentido, julga-se importante apresentar o que é entendido por emoção, a qual pode ser vista como um estado afetivo breve, de alta intensidade, como resposta sincronizada a um evento. Exemplos de emoções são: raiva, alegria, medo, etc (BERCHT, 2006). Já sentimento refere-se ao processo de viver uma emoção, "por 
definição, a experiência mental que nós temos do que se passa no corpo" (DAMÁSIO, 1996, p. 12).

Emoções e sentimentos são indispensáveis para a racionalidade, que não dependem apenas do cérebro, mas também da interação com o corpo. As emoções e os sentimentos são responsáveis por fazer previsões, planejar ações, e, assim, auxiliar nas decisões, "não são um luxo. Servem de guias internos e ajudam-nos a comunicar aos outros sinais que também os podem guiar" (DAMÁSIO, 1996, p.15).

Os cursos a distância, suportados por um AVEA, muitas vezes não proporcionam práticas de interação, e o feedback é automático. Assim, muitos estudantes sentem-se sozinhos, com pouca oportunidade de trocas sociais, sendo que a interação social também promove a aprendizagem. A afetividade assume o papel funcional na inteligência, que é utilizada como fonte energética necessária para o desenvolvimento da cognição (PIAGET, 2014). Dessarte, a Computação Afetiva apresenta possibilidades para potencializar a educação, promovendo motivação e acompanhando esse estudante que muitas vezes encontra-se sozinho no seu processo de construção de conhecimento, tendo em vista, que cada vez mais as modalidades de ensino e aprendizagem mediadas por meios virtuais são adotadas. Se os ambientes educacionais forem "munidos com mecanismos para reconhecer, mostrar emoções, certamente poderão flexibilizar estratégias de ação, providenciando suporte a aprendizagem de um estudante" (BERCHT, 2006, p.111).

Faz-se necessário superar a separação dos sujeitos presentes no processo de ensino e aprendizagem e dessa forma, oferecer interação, motivação, energia para que os estudantes se desenvolvam. Logo, uma maneira de promover a afetividade dentro de um AVEA é a utilização de Agentes Pedagógicos, os quais são caracteres visuais que residem no espaço digital dos sistemas virtuais de aprendizagem. São vistos como interfaces intrinsecamente sociais, o que permite a inclusão de valores socioemotivos no processo de aprendizagem (Guo e Goh, 2015; Kim e Baylor, 2015). Incorporado em AVEA, os Agentes Pedagógicos tomam funções instrutivas como tutor, companheiro de aprendizagem e instrutor (Kim e Baylor, 2015). Ou seja, o objetivo de Agentes Pedagógicos é auxiliar os aprendizes no seu processo de aprendizagem (BERCHT, 2001).

\section{3- Metodologia}

O presente estudo é caracterizado como uma Revisão Sistemática da Literatura (RSL), que é "um método para realizar o levantamento do estado da arte" (DERMEVAL et al, 2019). As questões norteadoras foram: Como ocorre a expressão de afetividade por Agentes Pedagógicos (AP) em Ambientes Virtuais de Ensino e Aprendizagem (AVEA)? A utilização de AP, que expressam afetividade, contribui para os processos educacionais ocorrido em AVEA?

A RSL aconteceu a partir da investigação de artigos publicados considerando como descritores de busca: (Affective Expression) AND Agent AND (Virtual Learning Environment). As palavras-chave foram definidas baseadas no objetivo de investigação do presente trabalho, isto é, como a expressão de afetividade dos AP em AVEA pode contribuir ou não no processo de aprendizagem dos alunos.

As buscas foram realizadas no Portal de Periódicos da Capes, Revista RENOTE e na International Conference on Advanced Learning Technologies. A escolha pelo evento e pela revista se deu por entender que há um espaço importante de publicações 
que permeiam o tema pesquisado, e a primeira base de buscas citada foi escolhida por tratar-se da maior fonte de buscas por trabalhos científicos no Brasil.

Delimitou-se o período entre 2014 e 2019, e, ainda, para refinar a pesquisa foram utilizados os filtros: I) periódicos revisados por pares; II) Artigos e III) tópicos: education; affective computing. Na CAPES o número de trabalhos encontrados foi de 78.

Nessa primeira investigação foi feita a leitura do título e palavras-chave, chegando ao resultado de 8 trabalhos selecionados. A mesma busca também foi realizada na revista Renote, compreendendo dez edições, onde foram selecionados 3 trabalhos. E, por fim, na International Conference on Advanced Learning Technologies, onde foram encontrados 5 trabalhos, a investigação foi realizada em cinco edições do evento, de 2014 a 2018, finalizando, assim, a primeira etapa com 16 trabalhos.

Na segunda etapa aconteceu a leitura dos resumos. Os critérios de exclusão foram: I) não trata de AP; II) não trata de afetividade; III) não trata de AVEA; IV) artigo de revisão. A partir dessa leitura, restaram 6 trabalhos ${ }^{1}$.

$\mathrm{Na}$ terceira etapa do presente estudo, uma leitura completa dos trabalhos selecionados foi realizada, dos quais foram excluídos mais dois trabalhos, pois foi verificado que não atendiam às definições já descritas nas etapas anteriores, dispondo, portanto, de 4 artigos para a análise e discussão.

Após a seleção dos trabalhos, com o intuito de responder às perguntas da presente Revisão Sistemática da Literatura, ocorreu a análise e discussões sobre os Agentes Pedagógicos de cada estudo e seus resultados, as quais são apresentadas na sequência.

\section{5- Resultados e Discussões}

Os trabalhos selecionados a partir dessa Revisão Sistemática da Literatura têm por objetivo encontrar maneiras, por diferentes caminhos, de contribuir para uma educação de melhor qualidade, diretamente ligadas ao processo de ensino e aprendizagem por meio de Agente(s) Pedagógico(s) dentro de um Ambiente Virtual de Ensino e Aprendizagem (AVEA).

O Quadro 1 elenca os trabalhos selecionados e sistematiza as especificidades de cada um, em relação aos Agentes Pedagógicos, suas características, expressão de afetividade e interação.

Quadro 1 - Análise dos Agentes Pedagógicos

\begin{tabular}{|c|c|c|c|c|}
\hline Trabalhos selecionados & $\begin{array}{c}\text { Liew et al } \\
\mathbf{( 2 0 1 6 )}\end{array}$ & $\begin{array}{c}\text { Lin, Wu, e } \\
\text { Hsueh (2014) }\end{array}$ & $\begin{array}{c}\text { Harley et al } \\
\text { (2016) }\end{array}$ & $\begin{array}{c}\text { Mudrick et } \\
\text { al (2017) }\end{array}$ \\
\hline Número de AP & 2 & 2 & 4 & 1 \\
\hline Características do AP & Humano & $\begin{array}{c}\text { Personagens que } \\
\text { representam } \\
\text { animais, um }\end{array}$ & $\begin{array}{c}\text { Personagens } \\
\text { que } \\
\text { representam }\end{array}$ & Humano \\
\hline
\end{tabular}

${ }^{1}$ Acredita-se que número baixo de trabalhos encontrados decorre de poucas investigações com esse foco específico, a partir disso, infere-se a importância em desenvolver trabalhos cuja abordagem seja a expressão de afetividade dos AP em AVEA.

$\mathrm{V} .1$ 'l $\mathrm{N}^{\mathrm{U}} 3$, dezembro, 2019 RENOTE DOI: 


\begin{tabular}{|c|c|c|c|c|c|}
\hline & & & gato e um sapo & humanos & \\
\hline & $\begin{array}{c}A c ̧ a \tilde{a} o \text { do } \\
A P\end{array}$ & $\begin{array}{c}\text { Expressão } \\
\text { facial } \\
\text { dinâmica } \\
\text { sincronizada } \\
\text { com a fala }\end{array}$ & $\begin{array}{c}\text { O AP informa o } \\
\text { aluno sobre o } \\
\text { status e } \\
\text { desempenho do } \\
\text { STA }\end{array}$ & $\begin{array}{c}\text { Expressão } \\
\text { facial, fala e } \\
\text { texto }\end{array}$ & $\begin{array}{l}\text { Expressão } \\
\text { facial }\end{array}$ \\
\hline & $\begin{array}{l}\text { Estados } \\
\text { afetivos }\end{array}$ & $\begin{array}{c}\text { Sorriso / } \\
\text { neutro }\end{array}$ & $\begin{array}{c}\text { Confuso, alegre, } \\
\text { chateado, } \\
\text { zangado, } \\
\text { entorpecido }\end{array}$ & $\begin{array}{l}\text { Prazer, } \\
\text { orgulho, tédio } \\
\text { e neutro }\end{array}$ & $\begin{array}{c}\text { Alegria, } \\
\text { confusão e } \\
\text { neutralidade }\end{array}$ \\
\hline $\begin{array}{c}\text { Como } \\
\text { ocorre a } \\
\text { expressão } \\
\text { de } \\
\text { afetividade }\end{array}$ & Meio & $\begin{array}{c}\text { AP } \\
\text { Animados } \\
\text { em 3D }\end{array}$ & $\begin{array}{c}\text { AP } \\
\text { Animados }\end{array}$ & AP Animados & $\begin{array}{c}\text { Gravações } \\
\text { em vídeo de } \\
\text { expressões } \\
\text { humanas } \\
\text { reais }\end{array}$ \\
\hline & $\begin{array}{c}\text { Momento } \\
\text { que atua }\end{array}$ & $\begin{array}{l}\text { O AP atua } \\
\text { em uma lição } \\
\text { interativa de } \\
30 \text { minutos } \\
\text { com os } \\
\text { alunos }\end{array}$ & $\begin{array}{l}\text { A partir do } \\
\text { reconhecimento } \\
\text { facial do aluno }\end{array}$ & $\begin{array}{c}\text { Sempre tem } \\
\text { um AP } \\
\text { visível na tela } \\
\text { do AVEA e } \\
\text { cada um é } \\
\text { responsável } \\
\text { por tarefas } \\
\text { específicas }\end{array}$ & $\begin{array}{c}\text { Quando o } \\
\text { estudante tira } \\
\text { dúvidas }\end{array}$ \\
\hline $\begin{array}{r}\mathbf{O A P} \mathbf{~ p} \\
\text { inter }\end{array}$ & $\begin{array}{l}\text { move } \\
\text { ío? }\end{array}$ & $\mathrm{NÃO}$ & SIM & SIM & SIM \\
\hline
\end{tabular}

Fonte: Autoras, 2019

O artigo de Liew et al (2016) teve como objetivo testar a hipótese de que uma expressão sorridente na face de um Agente Pedagógico falante poderia afetar positivamente as emoções, a motivação e os resultados de aprendizado de um estudante em um Ambiente Virtual de Ensino e Aprendizagem. No entanto, nesse trabalho, o AP não promove interação na sua relação com os alunos, pois sua expressão provoca uma percepção negativa, ou seja, a interação entre o AP e o aluno não teve forte impacto na aprendizagem a partir da exploração das emoções e motivações dos aprendentes. Nenhum dos participantes pensou que o agente virtual estava genuinamente expressando um estado de felicidade, a partir de seus relatos. Em vez disso, o sorriso do AP foi percebido como afiliativo /ser educado $(60,38 \%)$ ou dominante /escondendo emoções $(39,62 \%)$. 
Os resultados do estudo mostraram que as emoções provocadas pelo AP, ainda que com a intenção de motivar os alunos com o sorriso, podem não ter sido atingidas, provocando medo e/ou repulsa no aluno, os quais relataram a aparência artificial do AP como um potencial entrave de interação no AVEA. Salienta-se também, com base nos dados do referido trabalho, que não houve diferenças significativas de escores pós-teste entre os aprendizes em condições de sorriso e agente neutro. Assim, o desfecho de aprendizagem não foi afetado positivamente pela presença de um Agente Pedagógico sorridente. $\mathrm{O}$ estudo também permite refletir sobre a importância de desenvolver AP com emoções e expressões mais próximas das expressões humanas.

A contribuição do trabalho de Lin, Wu, e Hsueh (2014) se dá a partir do uso de STA (Sistema de Tutoria Afetiva), cujo objetivo foi apresentar instrução corretiva em disciplinas do curso de contabilidade, onde formou-se um mecanismo interativo humanizado pelo qual os Agentes Pedagógicos reconhecem e adaptam seu comportamento às emoções dos alunos e fornecem feedback adequado para tais. Nesse trabalho o STA inclui quatro módulos: reconhecimento afetivo (combina reconhecimento de emoções faciais e reconhecimento de emoções semânticas), agente tutor, conteúdo e estratégias de instrução para examinar a influência do STA na contabilidade de instruções corretivas sobre a eficácia da aprendizagem e usabilidade.

Os resultados da pesquisa revelaram que os benefícios de usar o STA para correção são a boa usabilidade do sistema e o alto desempenho de aprendizado. Assim, foi possível relacionar o uso do STA com a aprendizagem dos alunos, de maneira positiva, ou seja, o STA foi equipado com o modelo de reconhecimento de afeto, que permitiu aos usuários interagir com o sistema, e foi oferecido um agente tutor amável, o qual melhorou o senso de usuários de insegurança e negatividade que sentiram em relação ao uso do sistema pela primeira vez. Além disso, os participantes puderam contemplar as perspectivas futuras das aplicações e desenvolvimento do STA. A maioria deles tinha oferecido sugestões e expressado vontade de cursar outras disciplinas usando este sistema. Acredita-se que essa contribuição seja relevante, pois considera aspectos importantes sobre a afetividade para a área da educação, nesse caso a contabilidade, podendo ser expandido para outras áreas de conhecimento.

Todos os estudos foram selecionados por tratarem de expressão de afetividade por AP em AVEA, que é o foco desse trabalho, mas para além disso os AP do estudo de Harley et al. (2016) também reconhecem emoções, pois buscaram examinar as relações entre os traços de personalidade dos alunos, as emoções que tipicamente experimentam enquanto estudam e as que relataram experimentar na interação com os agentes. Os AP desse estudo são os seguintes: Sam, o estrategista, solicita aos participantes que se envolvam em estratégias de aprendizagem e assegura seu uso; Pam, a planejadora, estimula o planejamento (ativação de conhecimento anterior, definição de objetivos); Gavin, o guia, fornece orientação para os participantes no AVEA e administra avaliações de conhecimento pré-teste e pós-teste e medidas de auto-relato; Mary, a monitora, estimula e apoia os participantes em seus processos de monitoramento. Assim, os Agentes Pedagógicos têm o papel de otimizar o AVEA para diferentes tipos de aprendizes.

Os resultados demonstram que algumas características de emoções e traços de personalidade podem ser usados para prever as emoções dos alunos direcionadas a Agentes Pedagógicos específicos. Também fornecem sugestões para a adaptação de AP para apoiar a experiência de emoções adaptativas e minimizar a experiência em emoções não adaptativas dos alunos. O estudo de Harley et al (2016) trouxe a interação de quatro Agentes Pedagógicos afetivos, oferecendo uma análise detalhada, apresentando um método que os autores consideram de fácil implementação para criar 
ambientes de aprendizagem emocionalmente adaptáveis, baseados em agentes, e melhorar as interações entre os alunos e os Agentes Pedagógicos, a fim de apoiar a aprendizagem.

O estudo de Mudrick et al (2017) buscou examinar sistematicamente a influência da congruência da expressão facial do tutor, a relevância do conteúdo e o tempo de desempenho e emoção do aprendiz. Foram usadas gravações em vídeo de expressões humanas, justificadas por trazer a expressão real de um humano, nesse caso um ator, que é ainda mais treinado para tal tarefa. O AP teve o objetivo de fornecer suporte afetivo aos estudantes. $O$ tutor exibia uma expressão facial alegre quando o conteúdo na tela era totalmente relevante para a pergunta que o aluno estava tentando responder ou uma expressão facial confusa quando o conteúdo na tela era pouco relevante. $O$ MetaTutor foi o AVEA utilizado nesse estudo, projetado para examinar a influência de um agente tutor humano virtual nas estratégias de aprendizado cognitivo dos alunos, julgamentos metacognitivos e respostas emocionais durante o aprendizado.

Os resultados sugerem que o desempenho dos alunos é significativamente melhor quando um agente tutor humano expressa emoções que são congruentes com a relevância do conteúdo. E, também, que os alunos expressam significativamente mais confusão quando o agente tutor humano fornece expressões faciais incongruentes. A pesquisa contribuiu para criar uma base de evidências para a concepção de tutores humanos virtuais inteligentes e seus comportamentos para facilitar interações mais inteligentes entre aprendizes e agentes e influenciar a aprendizagem. Além disso, mostrou que congruência contextual e temporal são características importantes para o desenvolvimento de futuros Agentes Pedagógicos.

\section{5- Considerações Finais}

A partir do estudo desses quatro trabalhos foi possível refletir e reforçar a importância da afetividade na educação mediada por computador. Não se trata da resolução dos problemas em sua totalidade, mas os trabalhos mostram alguns caminhos que podem ser tomados e outros que não devem ser seguidos, a exemplo do estudo de Liew et al (2016), no qual a expressão do agente foi artificial e provocou medo nos estudantes.

Lin, Wu e Hsueh (2014) implementaram um sistema de tutoria afetiva com AP que, além de expressar afetividade também a reconhecia, o sistema ofereceu usabilidade e possibilitou o aprendizado dos estudantes. O estudo de Harley et al. (2016) também mostrou que para além da expressão de afetividade, o reconhecimento de emoções auxiliou na ação com o estudante para mediar o processo educacional. Por sua vez, o Agente Pedagógico do trabalho de Mudrick et al (2017), por meio de expressões faciais congruentes, possibilitou uma melhora significativa no desempenho dos alunos.

Dessa forma, é possível inferir que a utilização de Agentes Pedagógicos, que expressam afetividade, pode contribuir para os processos educacionais ocorridos em AVEA. Assim, sabendo do aumento da oferta e procura de educação mediada por computador, é importante buscar caminhos para que melhorias na educação aconteçam, apoiadas em dados científicos. Esses trabalhos contribuem cientificamente, pois apresentam dados consistentes que permitem direcionar esforços, possibilitando que seus resultados sejam utilizados para aplicação e avanços na área da Computação Afetiva. Enfim, trata-se de uma área apontada para contribuir com a ciência, podendo ser utilizada para aperfeiçoamento em diversas esferas.

Sabendo da importância da afetividade em todo o processo educativo, aqui direcionada à educação mediada por computador, essas discussões também podem 
auxiliar professores no que se refere à sua prática, auxiliando-os a identificar e considerar aspectos que são relevantes e podem contribuir na sua ação de ensinar, refletindo sobre o processo de aprendizagem do aluno.

Como limitação indica-se que apesar das bases utilizadas para busca indexarem uma grande quantidade de periódicos, ainda existem outros bancos de dados que podem agregar pesquisas para esse estudo.

\section{Referências}

BERCHT, M. Em direção a agentes pedagógicos com dimensões afetivas. Porto Alegre: UFRGS, 2001. Tese de Doutorado.

BERCHT, M.. Computação afetiva: vínculos com a psicologia e aplicações na educação. In: Conselho Regional de Psicologia de São Paulo. (Org.). Psicologia \& Informática: produções do III Psicoinfo e II jornada do NPPI. 1ed.São Paulo: Conselho Regional de Psicologia, 2006, p. 106-115.

DAMÁSIO, Antônio R., O erro de Descartes: emoção, razão e o cérebro humano/ Antônio R. Damásio; tradução portuguesa Dora Vicente e Georgina Segurado - São Paulo: Companhia das letras, 1996.

DERMEVAL, D.; COELHO, J. A. P. M.; BITTENCOURT, I. I. Mapeamento Sistemático e Revisão Sistemática da Literatura em Informática na Educação. In: JAQUES, P. A.; PIMENTEL, M.; SIQUEIRA; S.; BITTENCOURT, I. (Org.) Metodologia de Pesquisa em Informática na Educação: Abordagem Quantitativa de Pesquisa. Porto Alegre: SBC, 2019. (Série Metodologia de Pesquisa em Informática na Educação, v. 2) Disponível em: 〈https://metodologia.ceie-br.org/livro-2〉. Acesso em: Out/2019

FAHY, P. Characteristics of Interactive Online Learning Media. In: ANDERSON, T. (Ed.). The Theory and Practice of Online Learning. Athabasca: Athabasca University, 2008, p.167-200.

GUO, Y. R., \& GOH, D. H. L. Affect in embodied pedagogical agents meta-analytic review. Journal of Educational Computing Research, 53(1), 124-149, 2015.

HARLEY, J.; CARTER, C.; PAPAIONNOU, N.; BOUCHET, F.; LANDIS, R.; AZEVEDO, R.; KARABACHIAN, L. Examining the predictive relationship between personality and emotion traits and students' agent-directed emotions: toward emotionally-adaptive agent-based learning environments. User Modeling and User Adapted Interaction, Jun 2016, Vol.26(2-3), pp.177-219.

Kim, Y., \& Baylor, A. L. Research-based design of pedagogical agent roles: A review, progress, and recommendations. International Journal of Artificial Intelligence in Education, 26(1), 160-169, 2015.

LIEW, Tze Wei; ZIN, Nor Azan Mat; SAHARI, Noraidah; TAN, Su-Mae. The Effects of a Pedagogical Agent's Smiling Expression on the Learner's Emotions and Motivation in a Virtual Learning Environment. International Review of Research in 
Open and Distributed Learning, 2016, Vol.17(5), p.248-266.

LIN, Hao-Chiang Koong; WU, Chih-Hung; HSUEH, Ya-Ping. The influence of using affective tutoring system in accounting remedial instruction on learning performance and usability. Computers in Human Behavior, December 2014, Vol.41, pp.514-522.

MUDRICK, N. V.; TAUB, M.; AZEVEDO, R.; ROWE, J.; LESTER, j.. Toward affect-sensitive virtual human tutors: The influence of facial expressions on learning and emotion. Seventh International Conference on Affective Computing and Intelligent Interaction, 2017.

PIAGET, J. Relações entre a Afetividade e a Inteligência no Desenvolvimento Mental da Criança. Rio de Janeiro: Walk Editora, 2014.

PICARD, R. Affective Computing. Cambridge, Massachusetts: MIT Press, 1997. 292p. 